\title{
When Order Matters: Communication Scheduling for Current Injection Control in Micro Grids
}

\author{
Riccardo Bonetto, Stefano Tomasin, Michele Rossi \\ Department of Information Engineering (DEI) \\ University of Padova, Via G. Gradenigo 6/B, 35131 Padova (PD), Italy \\ \{bonettor, tomasin, rossi\}@dei.unipd.it
}

\begin{abstract}
We consider a smart micro grid where multiple distributed energy sources (DESs) inject current to support loads and reduce power losses over the lines. Distributed current control algorithms rely on a communication infrastructure, to exchange control data among DESs. For stability purposes, a token ring approach is often implemented for the control, where at any given time a single node with communication and control capabilities (smart node) has the token and is in charge of implementing the control action entailed by the algorithms (i.e., current injection). In this paper, we investigate the token scheduling, defining optimality criteria, devising lightweight suboptimal rules and assessing the performance of the optimal and suboptimal but online techniques. Two relevant power loss minimization schemes from the literature are considered to demonstrate the effectiveness of the proposed scheduling, which is assessed over a large number of grid topologies, that are statistically generated according to established literature models.
\end{abstract}

\section{INTRODUCTION}

We consider electricity grids where distributed energy sources (DESs) from renewables (e.g., photovoltaic panels or wind-powered micro turbines) exist and may act as energy producers to provide ancillary services. In the considered scenario, an overlay communication infrastructure [1] is utilized to orchestrate the DESs in a distributed fashion with the objective of minimizing power losses.

We note that the current flowing in the electrical transmission cables yields a partial power dispersion (in the form of heat) that contributes to economical and environmental costs. By suitably setting the amount of active/reactive power injected by DESs these power losses can be reduced, whilst sustaining the connected local loads. This translates into a decreased power demand to the mains, which lessens the use of high voltage lines and the associated operational cost for the utility.

Early works on the reduction of power losses focused on centralized solutions [2], [3], which are however hardly scalable as the grid size increases and new DESs are dynamically added. Moreover, they require a full knowledge of the grid, in terms of topology, load activity and DES power availability, thus also inducing a significant communication overhead. Distributed solutions were investigated in, e.g., [4][7]. While often being suboptimal, these are more flexible and have lighter requirements in terms of communication.

We observe that in the existing literature the communication infrastructure was often taken for granted and the communication patterns among nodes were not optimized. Most papers considered a sequential adjustment of the current injected from the smart nodes, i.e., at any given time, a single node updates the amount of current injected, while the remaining ones do not apply any change. This approach assures grid stability and induces a token-ring communication strategy where, at any given time, a single node has the token and implements the control action, requesting and sending data over the communication network. We stress that the order by which the token is passed among the nodes has not been considered in previous works, although this affects the convergence rate of the control algorithms as well as the power drained during the optimization process.

In this paper, we aim at optimizing the token exchange procedure among smart nodes in order to either reduce the token path length or to maximize the convergence rate of selected optimization algorithms for power loss minimization. Specifically, we investigate the importance of the scheduling rule that is utilized for the token assignment, assessing the impact of optimal control sequences as well as that of an original and lightweight heuristic approach. Two relevant power loss minimization algorithms, namely, the current based surround control (CBSC) [5], [8], and the distributed optimal reactive power flow control (DORPF) [6], [9], which have been proven to significantly reduce power losses in smart micro grids are considered. The performance of these schemes is then tested over a large number of grids that are statistically generated according to established literature models [10].

The rest of the paper is organized as follows. Section II presents the considered electrical grid model, and provides a short overview of the two selected control techniques. The optimization of the token assignment strategy is addressed in Section III. Details on the grid generation methodology are provided in Section IV, which is then used in Section V to obtain numerical results in terms of total dissipated power. Our concluding remarks are given in Section VI.

\section{SYSTEM MODEL}

In this section, we specify the electrical and communication models for the micro grid. In addition, we briefly review the selected optimization techniques, highlighting their communication requirements.

\section{A. Grid Model}

We model the micro grid electrical topology as a directed tree. The root of the tree represents the point of common coupling (PCC), the other nodes represent loads, distributed 


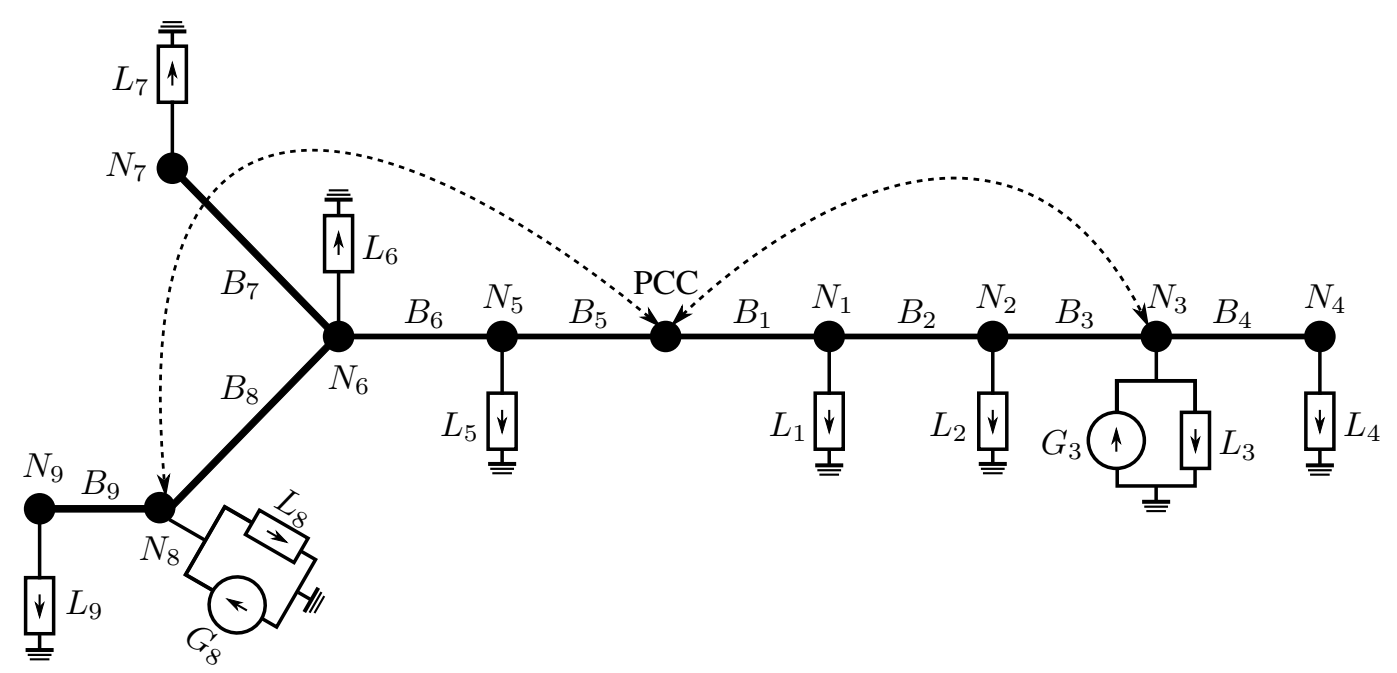

Fig. 1. Power micro grid example. Think lines represent line impedances, whereas dashed lines represent logical PLC communication links.

energy sources (DESs) and connection points. Loads are represented either as constant resistive-inductive series impedances or as constant current sources. DESs are modeled as current sources, which may be connected in parallel to a load, which is referred to as the associated load (DESs are always assumed to feed their associated loads). Some of the nodes are equipped with smart meters and powerline communication (PLC) transceivers and, in turn, are able to take electrical measures and to communicate using the power lines. These nodes are referred to as smart nodes (SNs) and are identified by indices $1, \ldots, N$ with $N$ being the total number of SNs. SNs identifiers are assigned by the PCC and remain fixed during the optimization. The communication capability of SNs induces a logical overlay communication network built on top of the power grid physical topology. The communication network is exploited to exchange local electrical measurements to minimize the distribution power losses and the total power demand to the PCC.

Fig. 1 shows a micro grid example with added communication capabilities. The set of nodes and branches are respectively denoted by $\mathcal{N}=\left\{\mathrm{PCC}, N_{i}: i=1, \ldots, 9\right\}$ and $\mathcal{B}=\left\{B_{j}: j=1, \ldots, 9\right\}$. Load $L_{i}$ is connected to node $N_{i}$ for $i=1, \ldots, 9$. DESs $G_{3}$ and $G_{8}$ are connected to nodes $N_{3}$ and $N_{8}$ and respectively feed the associated loads $L_{3}$ and $L_{8}$. In Fig. Fig. 1 the SNs are the PCC, $N_{3}$ and $N_{8}{ }^{1}$ The dashed lines connecting these SNs highlight the logical communication network structure, while communication data is exchanged over power cables thanks to PLC.

\section{B. Distributed Optimization Algorithms}

Local Control (LC) [4], [11] decreases the amount of power injected by the PCC by allowing DESs to directly feed their associated loads. This technique requires no communication among nodes and, in turn, the set of SNs is empty.

\footnotetext{
${ }^{1}$ Note that communication can only occur among smart nodes, as the remaining ones are not equipped with the required PLC communication capabilities.
}

Current Based Surround Control (CBSC) [5], [8] groups the nodes into clusters. Clusters are defined by checking, for any pair of DESs, whether their connecting path includes any other DES or the PCC. If this is not the case, a cluster is defined as the set containing the two DESs, the associated nodes, and all the nodes between them in the electrical network topology. For each cluster, the DES that is closest to the PCC is elected as the cluster head $(\mathrm{CH})$. In the case where one of the two DESs in the cluster is the PCC, this is elected as the $\mathrm{CH}$ (i.e., we assume that the PCC has better communication and computational resources with respect to the other nodes). Clusters in Fig. 1 are $C_{1}=\left\{\mathrm{PCC}, N_{1}, N_{2}, N_{3}\right\}$ and $C_{2}=$ $\left\{\mathrm{PCC}, N_{5}, N_{6}, N_{8}\right\}$. The DESs inject the current (complex or reactive depending on the optimization policy) that is required by the loads in the respective cluster. The current injected for optimization purposes is scaled by a real factor $0 \leq \alpha \leq 1$ such that, referring to $I_{C}$ as the total current needed in the cluster, the currents injected by the two DESs in the cluster are $\alpha I_{C}$ and $(1-\alpha) I_{C}$. The parameter $\alpha$ is determined for each cluster according to the instantaneous power demand from the loads therein and branch impedances. Hence, this technique requires that every node is a $\mathrm{SN}$.

Distributed Optimal Reactive Power Flow Control (DORPF) [6], [9] requires that DESs are grouped into possibly overlapping clusters and that, for each of them, one of the nodes becomes the cluster head $(\mathrm{CH})$. Also, within each cluster, the gradient of the power distribution loss is estimated through local measurements. Relying on the estimated gradient, the $\mathrm{CH}$ computes the set of reactive powers that have to be injected by the two DESs in its own cluster (one being associated with the $\mathrm{CH}$ ) in order to minimize the distribution power losses and spreads this information among its neighboring DESs. While different clustering procedures are possible, as stated in [6], the most effective clustering technique is the one proposed in [5] (see CBSC above). This technique requires that only the nodes that are connected to DESs are SNs, thus relaxing the requirements on the nodes in terms of communication and complexity. Due to this, the same 
clustering approach of CBSC is also considered for DORPF.

\section{TOKEN RING CONTROL}

CBSC and DORPF require that groups of nodes iteratively take a control action (i.e., inject a certain amount of power in the grid) in order to reduce as much as possible the distribution power loss. The PCC is considered as a SN during the optimization process and its identifier is 0 . The procedure of having at any given time a single SN allowed to modify the injected current, before letting the next $\mathrm{SN}$ to operate is similar to the token ring approach widely used in communication networks. Therefore, we will use here the related terminology, where however token ownership is associated to the current control, rather than to the possibility to transmit information. Indeed, when a node has the token it may communicate (in a two-way fashion) with other nodes in order to collect the information need for the control action. However, only SN with the token initiates the communications, while other nodes are only allowed to answer its requests. When the current token owner releases the token, the next owner is chosen according to a specific policy. Two policies for the owner selection are now discussed. The first ordering strategy aims at maximizing the convergence rate of the optimization algorithms. The second ordering strategy is considered as a comparison reference and simply aims at minimizing the length of the token path in each token round, i.e., minimize the communication overhead needed to move the token.

Heuristic for Convergence Rate Maximization: improving the convergence rate of the considered optimization algorithms has two main benefits. First, the optimization becomes more responsive to changes in the power demand from the loads. Second, further power is saved during optimization. The convergence rate can be improved by suitably tuning the order in which nodes perform the control action, i.e., defining a new token owner selection rule. The optimal (in the sense of maximum convergence rate) selection rule requires that at least one SN has a full knowledge of the network state, but, in this case, a centralized optimization approach would be the best choice. For this reason a heuristic selection rule, that does not increase the amount of information that each SN has to collect for the optimization purpose, is proposed. This rule is based on the observation that updates in clusters with a higher power demand should have a larger impact on the total power loss.

In details, any two clusters are referred to as adjacent if at least one pair of nodes belonging to the two clusters is connected by a line with no nodes in between. Hence, if a node belonging to the two clusters exists, the two clusters are adjacent. At the beginning of the optimization process, the token owner is uniformly chosen at random among the SNs by the PCC. At each optimization step, the token owner collects information about the actual power demand of all the adjacent clusters. The token is then passed to the head of the cluster with the highest power demand. If more $\mathrm{CHs}$ are eligible, one of them is chosen uniformly at random.

Token Path Length Minimization: as a baseline strategy we consider that obtained by minimizing the length of the token path. To this end, suitable SNs identifiers and a next owner updating rule have to be defined. The identifiers have to be assigned starting from 1 and visiting the nodes with a depth first pre-ordered tree traversal. If the current token owner is the SN with identifier $i$, then the next owner identifier will be $j=(i+1) \bmod N$. This procedure assures that the number of times the token has to jump between different subtrees is minimized, thus providing a more efficient communication solution, although in general suboptimal in terms of control algorithm convergence.

\section{Electrical Grid Topology Generation}

For a meaningful performance analysis, the selected optimization and scheduling algorithms are evaluated over a large number of power grid topologies. Toward this end, in this section a random power grid generation procedure, based on [10], [12] and used in [3], is briefly reviewed. According to [12], many real world networks can be successfully represented as small-world graphs. These graphs are generated starting from a regular ring lattice with $V$ vertices and degree $k$ (which are user defined parameters). The generation process considers each edge of the graph and, according to a user defined rewiring probability probability $p$, one of the endpoints of the edge is changed. The rewiring probability is a tunable parameter determining the degree of randomness of the generated graph. A zero rewiring probability leads to a completely regular graph, while a unitary rewiring probability leads to a completely random graph. Most real world scenarios are neither suitable to a completely regular representation, nor to a completely random one, thus an intermediate probability is often best suited to represent real networks.

In [10], it is pointed out that the small-world graph generation procedure does not account for some peculiar characteristics of real world power grids. In particular, actual grid topologies exhibit a quite low average degree, which would lead to disconnected graphs. For this reason, in this paper we adopt an ad hoc random graphs generation procedure based on small-world graphs. Specifically, starting from a tree with $V$ vertices, $V-1$ branches, and exactly one leaf, each branch of the tree is iteratively rewired to a new endpoint according to the rewiring probability $p$. This process is carried out while ensuring that the graph remains connected and that no loops are generated. The procedure ends when all branches have been visited. The length property of each branch is then generated according to an exponential distribution. Also, an increasing $p$ has two main effects on the generated graphs: the tree height is reduced and the maximum degree of the graph is increased. This implies that the PCC has a higher number of direct neighbor nodes as $p$ increases.

\section{Numerical Results}

In this section, the optimization algorithms of Section II are tested using the the two ordering (or token path selection) strategies of Section III over a large number of power grids, generated according to the procedure described in Section IV.

We considered networks with 15 and 50 nodes and two values of the rewiring probability: $p=0.3$ and $p=0.7$. Branch lengths are sampled (independently at random for 


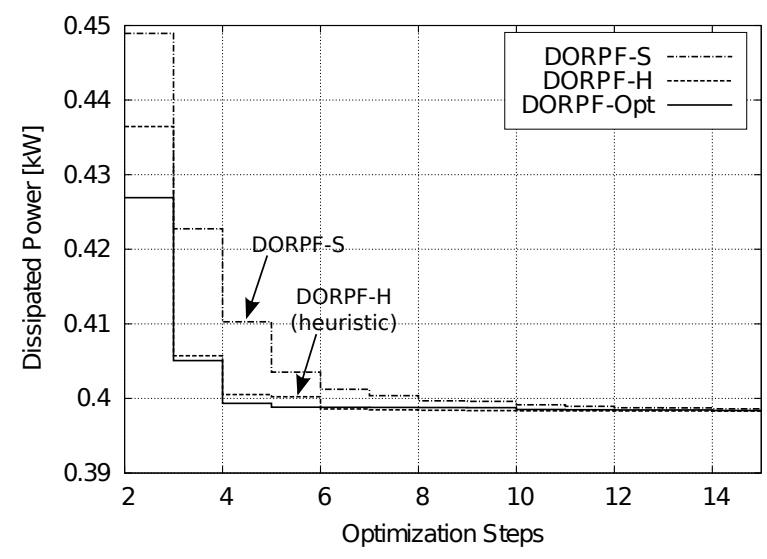

Fig. 2. Dissipated power $v s$ optimization steps for DORPF-S and DORPF-H and the power optimal strategy DORPF-Opt. 15 nodes, $p=0.3$.

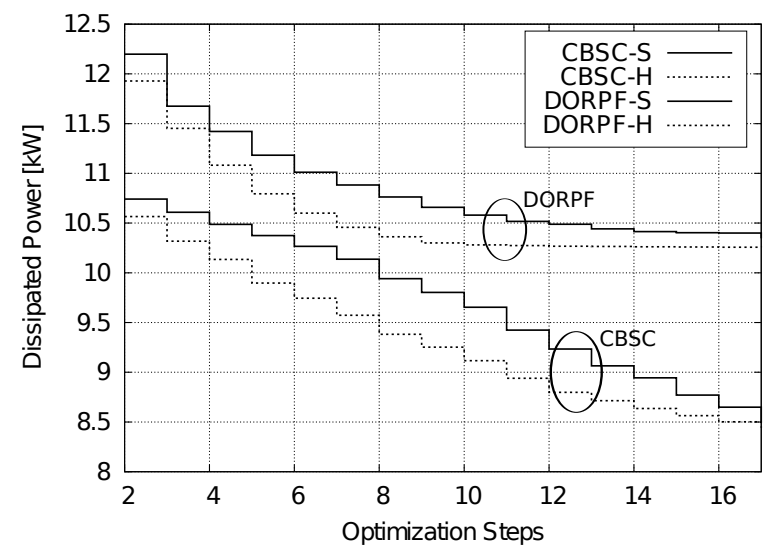

Fig. 3. Dissipated power vs optimization steps for CBSC-S, CBSC-H, DORPF and DORPF-H. 50 nodes, $p=0.3$.

each branch) from an exponential distribution with mean $\mu=100 \mathrm{~m}$. Power distribution cables are assumed to have constant section and, hence, constant impedance per meter. This impedance has been set to $(8+j 8) 10^{-6} \Omega / \mathrm{m}$. $30 \%$ of the nodes are connected to a DES. The nodes connected to DESs are chosen uniformly at random among the set of all nodes except the PCC. Each node (but the PCC) is connected to a load and loads are modeled as RL series impedances, whose values are chosen uniformly at random among the load types in Table I. The PCC imposes a voltage reference of $230 \mathrm{~V}$ and the grid frequency is $50 \mathrm{~Hz}$. The overlay communication network is assumed to be collision free. Moreover, it assumed that a routing protocol connecting each pair of SNs exists and that the communication links are error free.

TABLE I

RESISTANCE AND INDUCTANCE VALUES OF LOADS IMPEDANCES.

\begin{tabular}{|c|c|c|}
\hline Load type & $\mathrm{R}[\Omega]$ & $\mathrm{L}[\mathrm{mH}]$ \\
\hline LT1 & 8.79 & 12.7 \\
LT2 & 19.5 & 18.1 \\
LT3 & 3.39 & 8.1 \\
\hline
\end{tabular}

Next, we compare the two methods of Section III in terms of convergence rate and power expenditure during the

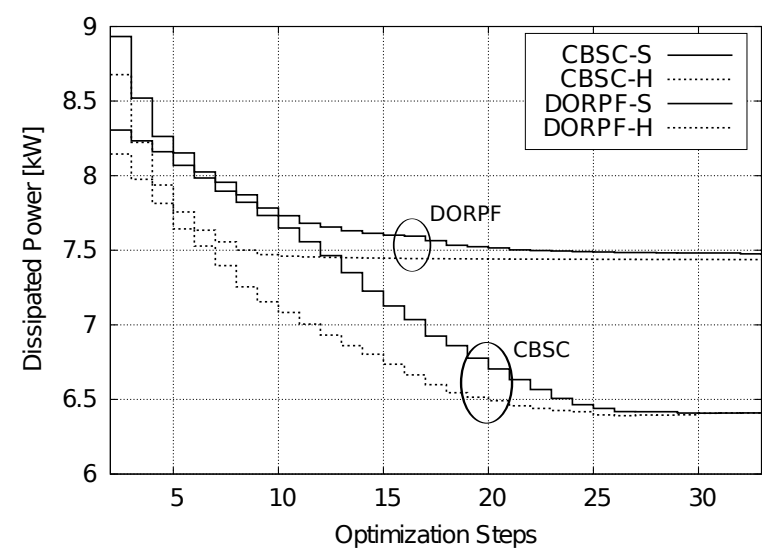

Fig. 4. Dissipated power vs optimization steps for CBSC-S, CBSC-H, DORPF and DORPF-H. 50 nodes, $p=0.7$.

optimization process. In the following, CBS and DORPF with the shortest token path policy will be denoted by CBSC-S and DORPF-S, respectively, while the same algorithms with the proposed heuristic for convergence rate maximization will be denoted by CBSC-H and DORPF-H, respectively.

Heuristic vs optimal scheduling: as a first set of results, in Fig. 2 we show the average power drained during the optimization process by DORPF-S, DORPF-H and an idealized version of DORPF, referred to here as "DORPF-Opt", that has been obtained by adopting the power optimal control sequence. The term optimal means that the sequence of nodes along the token path minimizes the energy drained during the execution of the algorithm. This optimal scheduling has been found through extensive search, an impractical approach whose complexity grows exponentially in the number of nodes and also requires full knowledge of electrical and communication topologies, DES and load (i.e., power demand) states. From Fig. 2, we see that our heuristic path selection (DORPF-H) performs very close to the power optimal scheme (DORPF-Opt), leading to gains in terms of convergence rate and energy expenditure.

Convergence rate: in Figs. 3 and 4 we compare DORPF against CBSC for $p \in\{0.3,0.7\}$ and increasing the number of nodes to 50. A first noticeable result is that the rewiring probability $p$ considerably affects the convergence rate of the considered optimization techniques. Specifically, from Fig. 3 we see that CBSC-S and DORPF-S converge within 17 optimization steps. For $p=0.7$ (see Fig. 4) the convergence rate remains almost constant for DORPF-S, while for CBSC-S about 10 additional optimization steps are required.

These results demonstrate that the convergence rate of the selected algorithms is sensitive to the grid topology. In detail, when the grid topology exhibits a low degree of randomness (i.e., $p=0.3$, Fig. 3 ), with the given setup, the maximum performance gap between CBSC-S and CBSC-H and between DORPF-S and DORPF-H is $0.5 \mathrm{~kW}$ and $0.4 \mathrm{~kW}$, respectively. As the degree of randomness increases (i.e., $p=0.7$, Fig. 4) the maximum gap between CBSC-S and CBSC-H rises to $0.7 \mathrm{~kW}$, while the maximum gap between DORPF-S and DORPF-H remains almost constant. However, DORPF- 
$\mathrm{H}$ converges in only 11 optimization steps, while DORPF-S takes approximately 30 optimization steps to converge. We observe that a higher $p$, in terms of electrical topology, means that nodes have a higher number of direct neighbors. This implies a much richer setting for the optimization, as a higher number of choices in terms of neighbor selection is available at each optimization step. The better performance of CBSC demonstrates that this algorithm, in spite of its simplicity, has a more efficient search strategy in the solution space and this comes at the expense of its longer convergence time. Note also that there are two main benefits arising from the adoption of our heuristic approach. The first benefit is that the power grid becomes more responsive to power demand variations due to a faster optimization phase (shorter convergence time). The second benefit is that, a faster convergence makes it possible to save a certain amount of energy during the optimization, as we discuss in greater detail below.

Energy savings: in Fig. 5 we show the complementary cumulative distribution function (CCDF) describing the probability of saving an amount of energy greater than or equal to the value in the abscissa when using CBSC-H or DORPF-H. This graph has been obtained for grid topologies with 50 nodes and $p=0.7$ by respectively integrating the power difference between CBSC-S and CBSC-H and between DORPF-S and DORPF-H for control steps of 1 minute each. For both CBSC and DORPF, the probability of saving energy during the optimization phase is greater than $90 \%$. When using CBSC-H, savings can be as high as $2 \mathrm{MJ}$ and this graph confirms the better optimization ability of this scheme. As an example, the probability of saving more than $0.5 \mathrm{MJ}$ is 0.4 for CBSC, whereas it is 0.2 for DORPF. Also, it is worth noting that a small number of grid topologies exist for which CBSC-H and DORPF-H converge slower than CBSC-S and DORPF-S, respectively, although they converge to the same final point, which is algorithmic dependent but only weakly dependent on the selected token path. While these topologies do not affect the average performance (shown in Fig. 3), their impact can be observed in Fig. 5. In fact, there is a small but positive probability that the energy gain provided by our heuristic scheduling is negative. Similar results, not shown here due to space constraints, are obtained for $p=0.3$.

To summarize, Fig. 5 shows that, for most of the grid topologies the heuristic approach proposed in this paper provides considerable energy savings during each optimization phase. In addition, since the optimization is repeated whenever the electrical grid state changes (especially in terms of variation of power demands) the cumulative gain is much higher and proportional to the rate at which the optimization algorithms are executed.

\section{Conclusions}

In this work we have analyzed optimal and heuristic scheduling rules to arbitrate the current injection from distributed energy resources in electricity grids. To this aim, we have considered two recent optimization schemes for the reduction of power distribution losses, discussing their communication requirements and comparing their performance

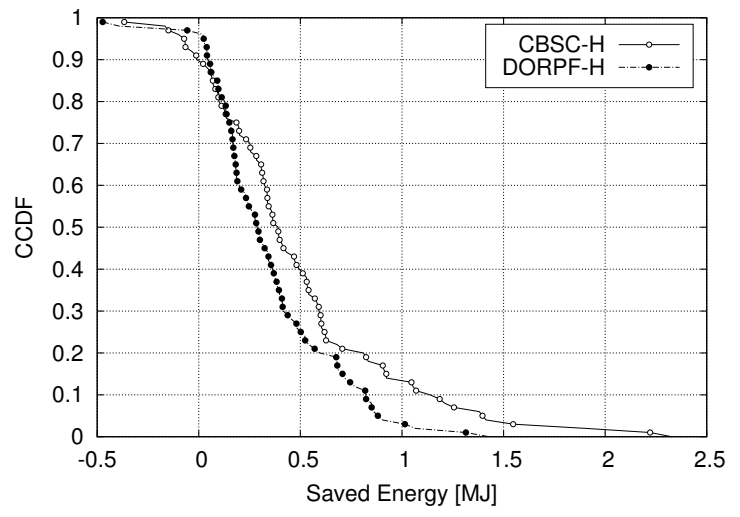

Fig. 5. CCDF of the energy saved by CBSC-H and DORPF-H with respect to CBSC-S and DORPF-S, respectively. 50 nodes, $p=0.7$.

against that of an idealized power optimal scheme. Our results reveal that the execution order (scheduling) for the distributed control actions matters and that substantial energy savings are possible though its careful design.

\section{REFERENCES}

[1] N. Bui, M. Rossi, and M. Zorzi, IEEE Vision for Smart Grid Communications: 2030 and Beyond. 3 Park Avenue New York, NY, USA: IEEE, 2013, ch. Networking Technologies for Smart Grid.

[2] A. Tsikalakis and N. Hatziargyriou, "Centralized control for optimizing microgrids operation," IEEE Trans. Energy Conversion, vol. 23, no. 1, pp. 241-248, March 2008.

[3] T. Erseghe and S. Tomasin, "Power flow optimization for smart microgrids by SDP relaxation on linear networks," IEEE Trans. Smart Grid, vol. 4, no. 2, pp. 751-762, Jun 2013.

[4] K. Turitsyn, P. Sulc, S. Backhaus, and M. Chertkov, "Local control of reactive power by distributed photovoltaic generators," in Proc. First IEEE International Conference on Smart Grid Communications (SmartGridComm), Gaithersburg, MD, U.S., Oct 2010.

[5] A. Costabeber, P. Tenti, and P. Mattavelli, "Surround control of distributed energy resources in micro-grids," in Proc. IEEE International Conference on Sustainable Energy Technologies (ICSET), Kandy, Sri Lanka, Dec 2010.

[6] S. Bolognani and S. Zampieri, "Distributed control for optimal reactive power compensation in smart microgrids," in Proc. 50th IEEE Conference on Decision and Control and European Control Conference (CDCECC), Orlando, FL, U.S., Dec 2011.

[7] A. Costabeber, T. Erseghe, P. Tenti, S. Tomasin, and P. Mattavelli, "Optimization of micro-grid operation by dynamic grid mapping and token ring control," in Power Electronics and Applications (EPE 2011), Birmingham, United Kingdom, Aug 2011, pp. 1-10.

[8] P. Tenti, A. Costabeber, P. Mattavelli, and D. Trombetti, "Distribution loss minimization by token ring control of power electronic interfaces in residential microgrids," IEEE Trans. Industrial Electronics, vol. 59, no. 10 , pp. 3817-3826, Oct 2012.

[9] S. Bolognani and S. Zampieri, "Convergence analysis of a distributed voltage support strategy for optimal reactive power compensation," in Proc. 3rd IFAC Workshop on Distributed Estimation and Control in Networked Systems, Santa Barbara, CA, U.S., Sept 2012.

[10] G. A. Pagani and M. Aiello, "Power grid network evolutions for local energy trading," arXiv:1201.0962 [physics.soc-ph], Feb 2012.

[11] K. Turitsyn, P. Sulc, S. Backhaus, and M. Chertkov, "Options for control of reactive power by distributed photovoltaic generators," Proceedings of the IEEE, vol. 99, no. 6, pp. 1063-1073, Jun 2011.

[12] D. J. Watts and S. H. Strogatz, "Collective dynamics of 'Small-World' networks," Nature 393, pp. 440-442, Apr 1998. 